\title{
EL DOCTOR MANUEL COBAS NÚÑEZ (1885-1956), PIONERO DE LA UROLOGÍA Y DE LA URO-RADIOLOGÍA EN GALICIA
}

\author{
L.A. FARIÑA PÉREZ \\ Urología. Centro Médico POVISA. Vigo (Pontevedra).
}

Actas Urol Esp. 28 (2): 89-94, 2004

\section{RESUMEN}

\section{EL DOCTOR MANUEL COBAS NÚÑEZ (1885-1956), PIONERO DE LA UROLOGÍA Y DE LA URO-RADIOLOGÍA} EN GALICIA

INTRODUCCIÓN: El desarrollo de la radiología urinaria con contrastes intracavitarios y endovenosos tuvo un papel esencial en la aparición de la Urología como especialidad, coincidiendo con otras circunstancias que la hicieron posible (la invención de instrumentos endoscópicos y de técnicas para el análisis de la orina y la función renal, los avances en la anestesia, la asepsia, el control de la hemorragia y la infección), y también necesaria (por la demanda social de estos servicios médicos especializados).

MATERIAL Y MÉTODO: Estudio de la vida y la obra del doctor Manuel Cobas Núñez (1885-1956), pionero en Galicia, con su práctica quirúrgica y sus publicaciones, de la urología y de la uro-radiología modernas.

RESULTADO: Después de formarse en el Hospital Necker y en el Instituto Rubio, Cobas fue uno de los primeros en dedicarse en Galicia a la urología, sobresaliendo por su interés en las enfermedades quirúrgicas del tramo urinario superior. Tuvo un conocimiento de la cirugía reno-ureteral muy destacado para la época. En agosto de 1931 publicó el libro "Pielografía ascendente y pielografía descendente", uno de los primeros y mejor documentado sobre la exploración del tramo urinario superior mediante pielografía retrógrada con catéter y pielografía endovenosa, escrito sólo unos meses después de que se comercializaran los contrastes endovenosos.

COMENTARIO: Trabajando en la práctica privada y al margen de los ámbitos académicos, Cobas es uno de los protagonistas de los inicios de la urología moderna gallega. Sus publicaciones y su libro sobre la pielografía le muestran como uno de los primeros expertos en cirugía renal y también debe ser reconocido como pionero en Galicia y en España de la radiología de interés urológico.

PALABRAS CLAVE: Historia de la Urologia. Historia de la Uro-radiología.

\section{ABSTRACT}

\section{DOCTOR MANUEL COBAS NÚÑEZ (1885-1956), PIONEER OF UROLOGY AND URORADIOLOGY} IN GALICIA (SPAIN)

INTRODUCTION: The development of urologic radiology with intracavitary and intravenous contrast agents played an essential role in the rise of Urology as a surgical speciality, in agreement with other circunstances that made it both possible (the invention of endoscopic instruments and laboratory technics to study the urine and the kidney function, advances in anaesthesia, asepsia, control of haemorraghe and infection), and necessary (because of social request for these specialised medical services ).

MATERIAL AND METHOD: Study of the life and work of Manuel Cobas Núñez (1885-1956), whose surgical practice and publications pioneered the modern Urology and Uroradiology in Galicia (Spain).

RESULT: After being trained in Necker Hospital (Paris) and Instituto Rubio (Madrid), Cobas was one of the firsts to practice urology in Galicia, with a high interest in surgical diseases of the upper urinary tract. His knowledge of renoureteral surgery was outstanding for the time. In august 1931 he published "Pielografia ascendente y pielografia descendente", one of the firsts and best documented books on upper urinary tract examination using retrograde and intravenous pyelography, written only months after marketing of the first intravenous contrasts agents.

COMMENT: Working in private practice, out of the academic fields, Cobas was a protagonists of the beginning of Galician modern urology. His publications and book on pyelography show that he was one of the first experts in renal surgery and must be also recognized as a pioneer of uroradiology in Galicia and Spain.

KEY WORDS: History of Urology. History of Uroradiology. 


\section{INTRODUCCIÓN: LA UROLOGÍA MODERNA EN GALICIA}

En el primer tercio del siglo XX, no existen en Galicia urólogos con una práctica monográfica, pero, como en otros países europeos, se dedican a las vías urinarias, cirujanos con interés en el aparato urinario, ginecólogos que conocen la cirugía vaginal, y dermato-venereólogos especializados en la sífilis, la gonococia y sus complicaciones uretrales. Estos últimos serán los iniciadores de los primeros Servicios de Vías Urinarias, Piel y Venéreas (principalmente Manuel Villar y Manuel Pereiro, en Santiago y Nicolás Peña en Vigo), mientras que los primeros realizan la cirugía urogenital más compleja de entonces (de los genitales y uretra, la adenomectomía en dos o en tres tiempos, la litiasis vesical y raramente renal). Todos ejemplifican con su forma de actuar sobre el área urogenital, lo que en otros países y en algunas ciudades españolas ya venía conociéndose como cirugía urogenital o urología.

Coincidiendo con esta inicial actividad quirúrgica hecha en el ámbito de la beneficencia y en los hospitales universitarios, se crearon los primeros Sanatorios particulares, donde también se realizaban intervenciones de cierta complejidad, un signo de los cambios en las formas de atender la enfermedad que trajo el nuevo siglo, con los avances en el diagnóstico de las enfermedades, la cirugía y la anestesia. Por otra parte, el desarrollo de la radiología del tramo urinario con contrastes intracavitarios y endovenosos tuvo un papel esencial en la aparición de la Urología como especialidad en Europa y América, coincidiendo con otras circunstancias que la hicieron posible (la invención de instrumentos endoscópicos y de técnicas de laboratorio para el análisis de la orina y la función renal; la aparición y avances rápidos en la anestesia, la asepsia, el control de la hemorragia y de la infección); y también que la hicieron necesaria, por la demanda social de estos servicios médicos especializados ${ }^{1}$.

Las actividades de algunos pioneros de la urología que trabajaron fuera de los centros académicos, contribuyeron en buena medida tanto al desarrollo de la cirugía urológica como a la aplicación de la radiología al estudio de las enfermedades del tramo urinario, poniendo las bases de una especialidad que luego se haría pujante como una rama de la cirugía.

\section{EL DOCTOR MANUEL COBAS NÚÑEZ: BIOGRAFÍA}

El doctor Manuel Cobas Núñez (Fig. 1) fue en Galicia uno de los primeros en dedicarse a una práctica urológica moderna, que inicialmente simultánea con la medicina general en la comarca de Vigo, destacando sobre todo por su interés en las enfermedades quirúrgicas del tramo urinario superior. Nacido el 25 de agosto de 1885 en Meira (Pontevedra) de una familia humilde, estudia en el Instituto de Pontevedra y a continuación simultánea las licenciaturas de Medicina y Farmacia en Santiago. Trabaja unos años como médico general y posteriormente se traslada a Madrid, donde se forma en medicina y cirugía urológica en el Instituto Rubio, con Luis González Bravo, y en el Hospital Necker de París, que entonces dirigía Félix Legueu (Fig. 2). Defiende en Madrid en 1915 su tesis "Tratamiento endoscópico de los tumores de la vejiga". A su regreso a Vigo, en 1920, continúa su actividad como médico generalista e instala su consulta particular

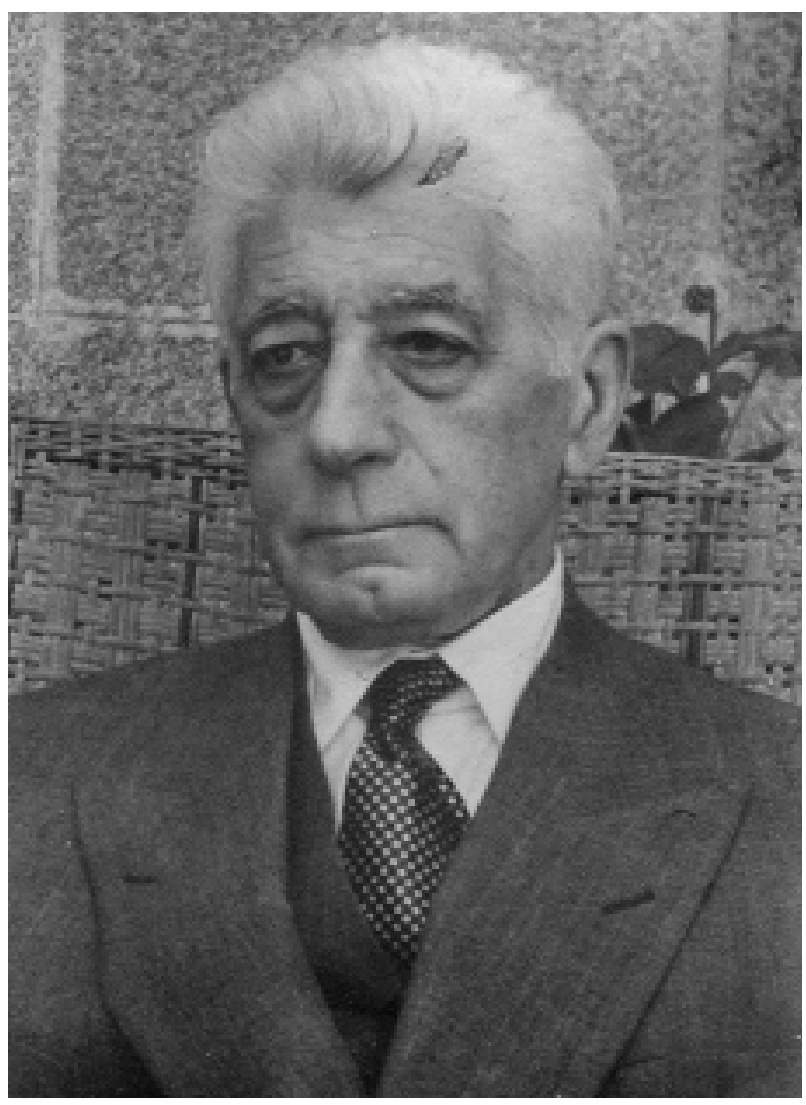

FIGURA 1. Manuel Cobas Núñez (1885-1956). 


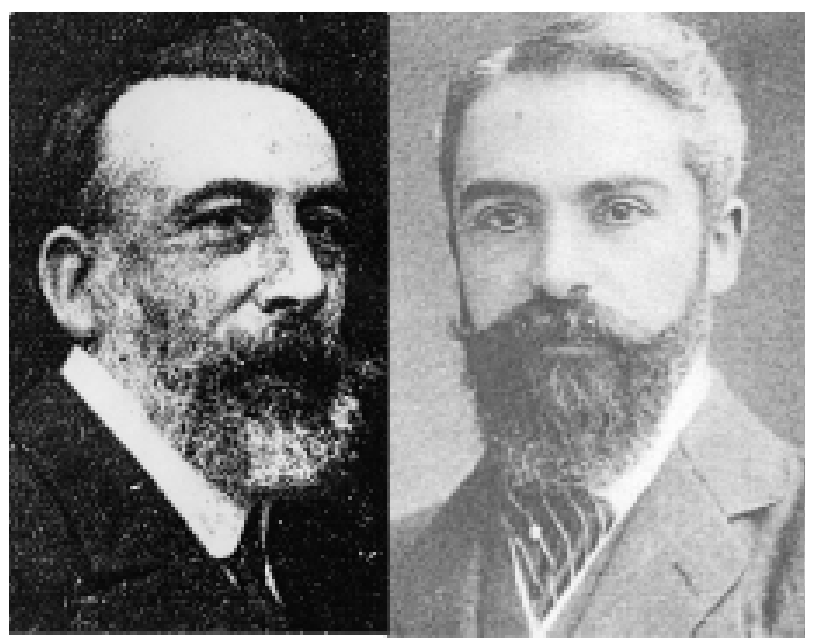

FIGURA 2. Luis González Bravo y Félix Legueu, maestros de Cobas en Madrid y Paris.

como urólogo, con un moderno aparataje radiológico. Socio fundador de la Academia MédicoQuirúrgica de Vigo, y su presidente de 1933 a 1936, desarrolló una intensa labor docente entre los médicos vigueses, divulgando la enfermedad litiásica y la cirugía reno-ureteral, tanto en trabajos publicados en el Boletín del Colegio Médico de Pontevedra como en los Anales de la Academia Médico-Quirúrgica de Vigo, o en sus comunicaciones a la misma Academia. Sus trabajos, con títulos como "Anomalías reno-ureterales", "Pionefrosis calculosa y fístula uropurulenta espontánea", "Fisiopatología del aparato excretor del riñón", "Litiasis reno-ureteral", "Bases del tratamiento de la litiasis reno-ureteral", "Discusión etiopatogénica de un caso de anuria de 13 días de evolución", muestran un conocimiento de la cirugía urológica y una habilidad y decisión quirúrgica muy destacados para la época. Nicolás Peña, que fue desde 1940 jefe del Servicio dermatovenereológico y de vías urinarias del Hospital Municipal de Vigo (actual Hospital Nicolás Peña), pero tenía poca inclinación por la cirugía, reconoce en alguno de sus textos que las intervenciones más comprometidas eran realizadas en esos años por Manuel Cobas.

En agosto de 1931, Cobas publica el libro "Pielografía ascendente y pielografía descendente", impreso en el establecimiento tipográfico "Faro de Vigo"2 (Fig. 3). Es uno de los primeros, mejor editado y documentado sobre el tema de la exploración radiológica del tramo urinario supe-

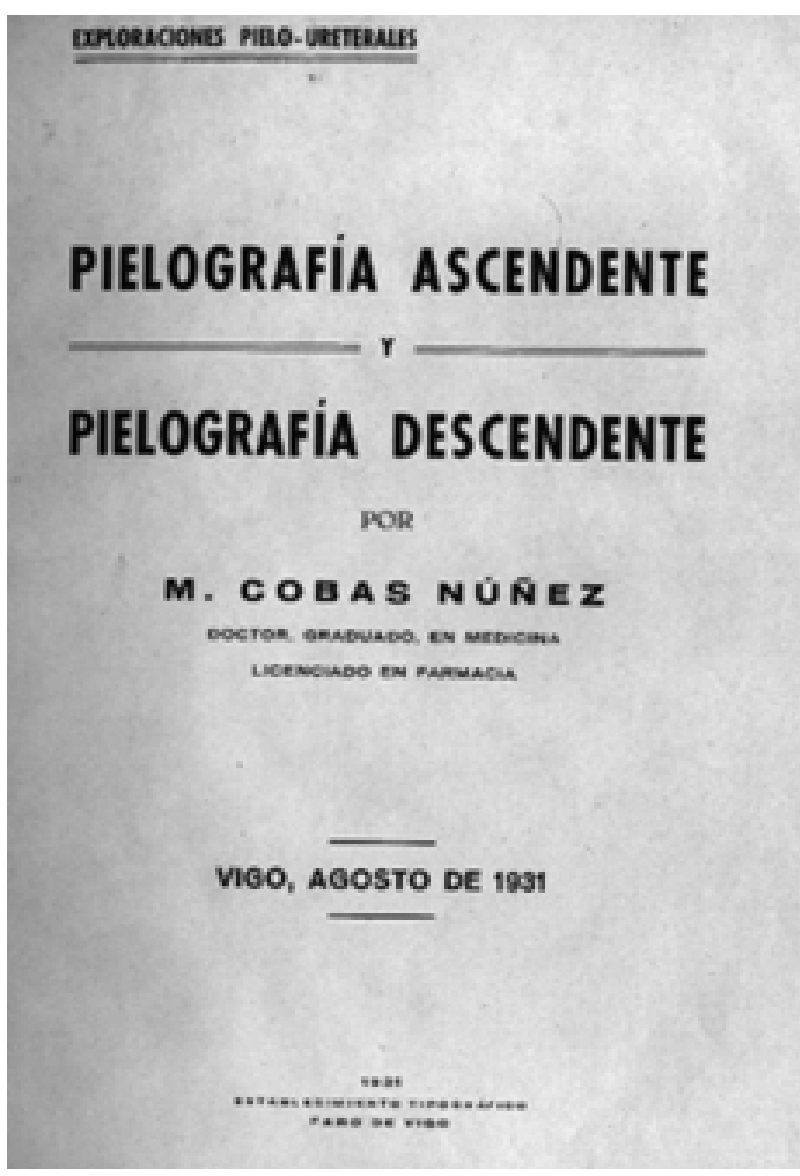

FIGURA 3. Portada del libro "Pielografia ascendente y pielografia descendente" (1931).

rior mediante pielografía retrógrada con catéter y pielografía endovenosa, y está escrito sólo unos meses después de la comercialización de los primeros contrastes endovenosos, lo que muestra el interés y actualización del autor sobre esta materia, heredada de su formación con los maestros franceses de Necker, el profesor Legueu, y sus jefes de clínica y radiología, Fey y Truchot. También es quizá la primera obra de su género en castellano, pues su información sobre la pielografía "descendente" o endovenosa se adelanta en unos años a las publicaciones realizadas en Madrid, Barcelona e Hispanoamérica, con excepción de algunos trabajos presentados en congresos o publicados en revistas por urólogos como Pedro Cifuentes ${ }^{3}$, los hermanos de la Peña ${ }^{4}$, Pascual $^{5}$, Puigvert ${ }^{6}$ o Gil-Vernet ${ }^{7}$. Los primeros contrastes endovenosos de uso clínico seguro, Uroselectán y Abrodil, habían comenzado a usarse en 1929 y se publicitaron en el Congreso 
Internacional de Urología celebrado en Madrid en 1930. El "Atlas de Urografia" de A. Puigvert, tal vez el mejor libro de este tiempo sobre urografía en lengua castellana, se publicó en el año 1933 y se agotó el mismo año, siendo una obra de referencia en Europa por presentar de forma extensa una relación entre los hallazgos anatomo-clínicos y los radiológicos en las enfermedades urinarias, una experiencia que Puigvert había comenzado en el año $1928^{8}$. Puigvert conocía bien el libro de Cobas, que figuraba en su biblioteca y está incluida entre las referencias bibliográficas de su "Atlas". Sin embargo, Pedro Gausa en "La pielografía en vertical en las hidronefrosis", de $1944^{9}$, no hace referencia al texto de Cobas, aunque sí cita los trabajos de Puigvert de 1934 y de L. de la Peña de 1924. Lo más probable es que un libro en una edición modesta, publicado en una región española que en aquellos años no tenía ningún peso en el ámbito urológico, tuviera una influencia muy limitada. No hay datos que permitan afirmar que este libro se conociera en otros países europeos o hispano-americanos, pues no aparece referenciado en las obras sobre el tema publicadas en aquellos años.

\section{EL LIBRO "PIELOGRAFÍA ASCENDENTE Y PIELOGRAFÍA DESCENDENTE "}

El libro se divide en una introducción, dos partes dedicadas a la pielografía ascendente (o retrógrada) y descendente (o urografía endovenosa), con estudio de las relaciones clínico-radiológicas y presentación de casos clínicos; conclusiones y bibliografía. En la Introducción, en un tono sin pretensiones, reconociendo la humildad de su práctica y la ausencia de referencias doctrinales propias, hace un repaso histórico de los orígenes de la aplicación de los rayos $\mathrm{X}$ al diagnóstico de los cálculos urinarios y de otras enfermedades del riñón, que es también una revisión del conocimiento de las enfermedades renales, médicas y quirúrgicas, hasta que las enseñanzas de Guyon y Albarrán dan origen a la especialidad urológica. Cobas demuestra tener conocimientos enciclopédicos sobre los esfuerzos realizados en Europa y América para lograr estudios radiológicos fiables del tramo urinario superior, hasta disponer de sustancias seguras que administradas por vía venosa proporcionan buenos pielogramas (pielografía descendente). Su propia experiencia con la pielografía descendente data de mayo de 1930, pocos meses después de la comercialización de los primeros contrastes endovenosos (Fig. 4). La primera parte del libro contiene los siguientes capítulos:

1. Parte general: historia, técnica, medios y complicaciones de la pielografía ascendente; 2 . Anatomía y fisiología de la pelvis y del uréter; 3. Parte especial: pielitis-pielonefritis, tuberculosis renal, riñón móvil, hidronefrosis, tumores del riñón, anomalías reno-ureterales, litiasis renoureteral.

$\mathrm{Y}$ en la segunda parte:

4. Parte general: pielografia descendente. Tipos de contrastes. Técnica y ejemplos de pielogramas con Uroselectan y Abrodil; 5. Parte especial: pielonefritis, pionefrosis, tuberculosis renal, riñón movible, anomalías del riñón y del uréter, hidronefrosis.

En las Conclusiones, Cobas reconoce en un momento muy temprano el valor, que en los años siguientes será enorme, de la pielografía descendente para los casos en los que la exploración transuretral, y por tanto la pielografía ascendente, es imposible. La tuberculosis renal sería el mejor ejemplo en el que la pielografía descendente era muy superior a la ascendente. Señala además que se trata de una exploración asequible a cualquier médico sin conocimientos o medios de exploración vesical. Destaca que hace posible el estudio simultáneo de ambos riñones, la exploración es más fisiológica que el examen ascendente, pues no se inyecta contraste a presión, y se reproduce la función excretora renal normal.

\section{COMENTARIO}

En el primer tercio del siglo XX había una alta prevalencia en toda Europa de dos enfermedades renales, la tuberculosis y la litiasis, cuyo estudio y tratamiento, junto al de las enfermedades del tramo urinario inferior que se conocían (sobre todo el adenoma de próstata, la estenosis de uretra y la litiasis vesical), inducirán la especialización de algunos cirujanos en las competencias anatomo-quirúrgicas del tramo génito-urinario, la invención de instrumentos endoscópicos, el desarrollo de la radiología y de técnicas de labo- 

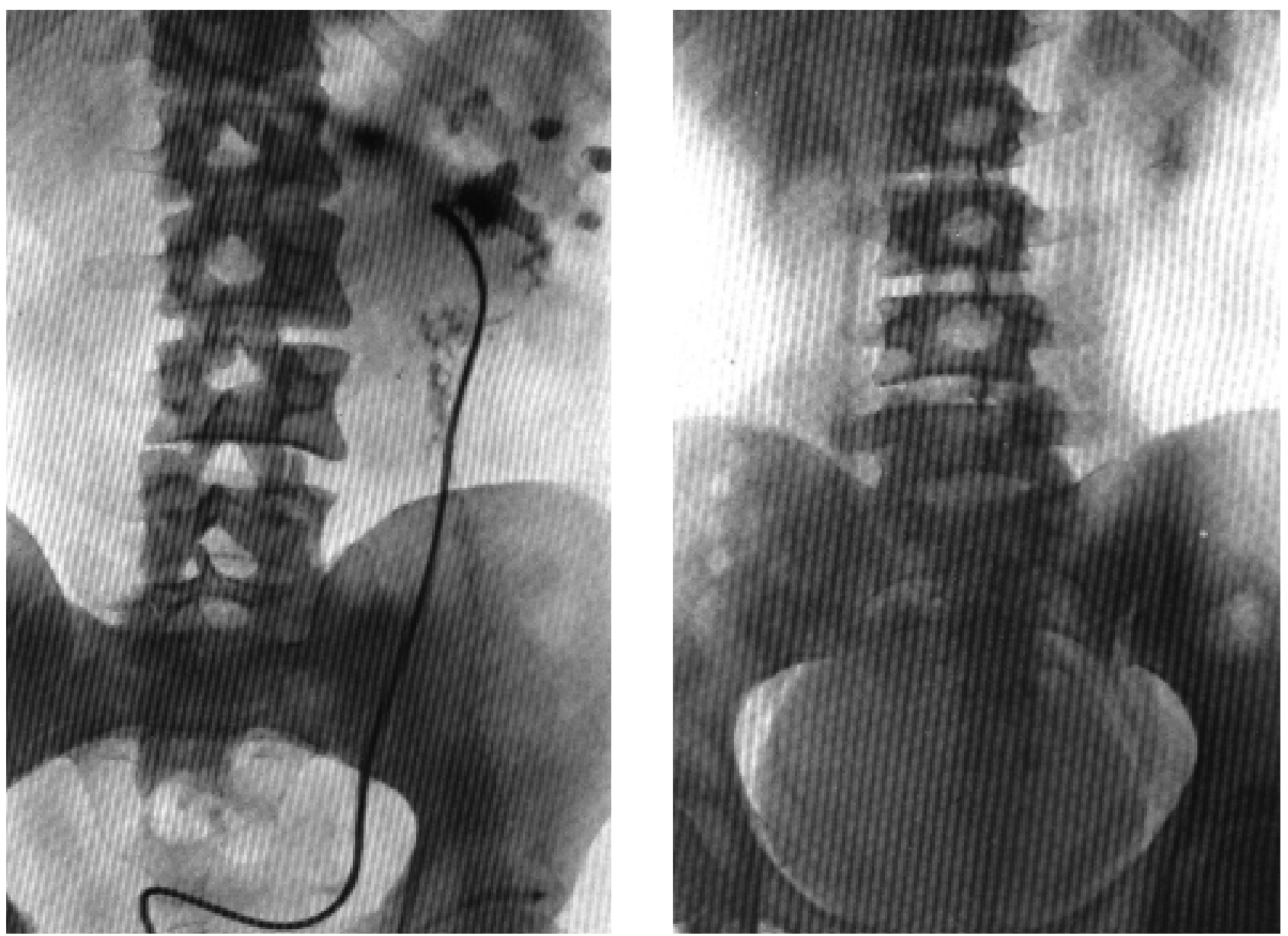

FIGURA 4. Dos de las figuras del libro en las que se aprecia la calidad de las exposiciones radiológicas hechas por Cobas. Pielografia retrógrada en una pionefrosis con fistula urinaria (izquierda). Pielograma endovenoso con Uroselectán (derecha).

ratorio para medir la función renal y analizar la orina. Todas estas circunstancias darán lugar, con la concurrencia imprescindible de la anestesia, de la antisepsia y finalmente de los antimicrobianos y de la transfusión sanguínea, a la especialización urológica tal como lo conocemos hoy, perfilada de manera paralela a casi todas las demás vertientes del panorama quirúrgico actual.

El desarrollo de la exploración radiológica con contrastes de la vía urinaria ${ }^{10-12}$, a la que contribuyeron médicos por entonces especializados en diversas áreas (dermatología, química, urología, radiología), tuvo una aplicación especialmente fructífera en los ámbitos que estaban en Europa "inventando" la urología, particularmente en Francia y Alemania, y contribuyó de manera significativa al mejor conocimiento de las enfermedades de las vías urinarias. Sus ventajas fueron tan rápidamente vistas que se difundió con gran rapidez.

Manuel Cobas Núñez, formado en Madrid y en el Hospital Necker de París al lado de Félix Legueu, y que trabajó como urólogo en Vigo desde los años 20, es un precursor en Galicia de la generación de urólogos modernos, que desarrollarán la especialidad a partir de los años 50, después de formarse en las escuelas más pujantes de Madrid y Barcelona. Trabajando en la práctica privada y al margen de los ámbitos académicos, Cobas es uno de los más interesantes protagonistas de los inicios de la urología moderna gallega. Sus publicaciones en revistas y su libro sobre la pielografía le muestran como uno de los primeros expertos en cirugía renal y también debe ser reconocido como pionero en Galicia y en España de la radiología de interés urológico. 


\section{REFERENCIAS}

1. FARIÑA LA.: Historia de la Urología moderna en Galicia y en Asturias. En: Maganto E. y Pérez Albacete M., eds: Historia biográfica y bibliográfica de la Urología española en el siglo XX. Edimsa. Madrid 2002: 203-218.

2. COBAS NÚÑEZ M.: Pielografía retrógrada y pielografía anterógrada. Faro de Vigo. Vigo, 1931.

3. CIFUENTES P.: La pyélographie par injection intraveineuse dans la tuberculose rénale. $J$ d'Urol (Paris) 1929; 30: 583.

4. DE LA PEÑA L, DE LA PEÑA E.: Nuestra experiencia actual sobre la pielografía intravenosa. Archivos de Medicina, Cirugía y Especialidades (Madrid), julio 1930.

5. PASCUAL S.: La pielografía intravenosa. Medicina Ibera (Madrid), junio 1930.

6. PUIGVERT A.: La urografía por los aceites yodados. Revista Médica de Barcelona 1929; 12: 426.

7. GIL-VERNET S.: Resultados de las primeras radiografías con Abrodil. Ars Médica 1930: 26.

8. PUIGVERT A.: Atlas de Urografía. Salvat. Barcelona, 1933.
9. GAUSA P.: La pielografía en vertical en las hidronefrosis. Miguel Servet. Barcelona, 1944.

10. GRAINGER RG.: The historical development of intravascular radiological contrast agents. En: Thomas AMK, ed. The invisible light. 100 years of medical radiology. Blackwell Science. Oxford 1995: 34-38.

11. LOHR E.: Urinary tract. En: Rosenbusch G, Oudkerk M, Ammann E., eds. Radiology in medical diagnosis. Evolution of X-ray applications 18951995. Blackwell Science. Oxford, 1995: 163-175.

12. ELKIN M.: Stages in the growth of uroradiology. Radiology 1990; 175: 297-306.

Dr. L.A. Fariña

Servicio de Urología

Centro Médico POVISA

C/ Salamanca, 5

36211 Vigo (Pontevedra)

(Trabajo recibido el 11 julio de 2003) 